\title{
Zuo Jin Wan Reverses DDP Resistance in Gastric Cancer through ROCK/PTEN/PI3K Signaling Pathway
}

\author{
Meng-Yao Sun, ${ }^{1}$ Jian Sun, ${ }^{2}$ Jie Tao, ${ }^{1}$ Yu-Xia Yuan, \\ Zhen-Hua Ni $\mathbb{D}^{1}{ }^{1}$ and Qing-Feng Tang $\mathbb{1}^{1}$ \\ ${ }^{1}$ Department of Clinical Laboratory and Central Laboratory, Putuo Hospital, Shanghai University of \\ Traditional Chinese Medicine, Shanghai 200062, China \\ ${ }^{2}$ Department of Clinical Laboratory, Shuguang Hospital, Shanghai University of Traditional Chinese Medicine, \\ Shanghai 200021, China
}

Correspondence should be addressed to Qing-Feng Tang; tangqingfeng126@126.com

Received 24 June 2018; Accepted 22 November 2018; Published 2 December 2018

Academic Editor: Siyaram Pandey

Copyright (C) 2018 Meng-Yao Sun et al. This is an open access article distributed under the Creative Commons Attribution License, which permits unrestricted use, distribution, and reproduction in any medium, provided the original work is properly cited.

\begin{abstract}
Gastric cancer (GC) is the third leading cause of cancer-related death. Chemotherapy resistance remains the major reason for GC treatment failure and poor overall survival of patients. Our previous studies have proved that Zuo Jin Wan (ZJW), a traditional Chinese medicine (TCM) formula, could significantly enhance the sensitivity of cisplatin (DDP)-resistant gastric cancer cells to DDP by inducing apoptosis via mitochondrial translocation of cofilin-1. However, the underlying mechanism remains poorly understood. This study aimed to evaluate the effects of ROCK/PTEN/PI3K on ZJW-induced apoptosis in vitro and in vivo. We found that ZJW could significantly activate the ROCK/PTEN pathway, inhibit PI3K/Akt, and promote the apoptosis of SGC-7901/DDP cells. Inhibition of ROCK obviously attenuated ZJW-induced apoptosis as well as cofilin-1 mitochondrial translocation, while inhibition of PI3K had the opposite effects. In vivo, combination treatment of DDP and ZJW (2000 mg/kg) significantly reduced tumor growth compared with DDP alone. Moreover, the combined administration of ZJW and DDP increased the expression of cleaved ROCK and p-PTEN while it decreased p-PI3K and p-cofilin-1, which was consistent with our in vitro results. These findings indicated that ZJW could effectively inhibit DDP resistance in GC by regulating ROCK/PTEN/PI3K signaling and provide a promising treatment strategy for gastric cancer.
\end{abstract}

\section{Introduction}

GC is one of the most prevalent cancers characterized by high morbidity and mortality [1]. Surgery remains the only curative therapeutic methods so far. However, due to its late disease presentation, GC was detected in most of the patients at an advanced stage when the tumor is usually migrated, causing an extremely low 5-year survival rate [2]. For advanced gastric cancer, chemotherapy is the preferred therapeutic strategy. DDP is considered as a common drug for the treatment of GC [3]. DDP based adjuvant chemotherapy has been approved to increase survival after gastric resection [4]. Unfortunately, intrinsic or acquired drug resistance seriously limits the treatment effect of DDP [4]. Therefore, it is necessary to develop effective strategies to increase the sensitivity of DDP in GC treatment.
ZJW, a traditional Chinese medicine formula, composed of Rhizoma Coptidis (Huanglian in China) and Evodia Rutaecarpa (Wuzhuyu in China) with ratio of $6: 1$, has been used to treat gastrointestinal diseases in China for a long time and showed better therapeutic effects in adjuvant treatment of tumors [5-7]. Our previous studies have demonstrated that ZJW could significantly enhance the sensitivity of DDPresistant gastric cancer cells to DDP and executed their biological effects by increasing mitochondrial apoptosis via PP1 and PP2A induced the dephosphorylation of p-cofilin1, which implied that ZJW might serve as a synergistic drug with DDP in the treatment of gastric cancer [7]. However, it remains unclear how ZJW inhibits the DDP resistance in gastric cancer cells involving the modulation of cofilin-1 activity.

It is well known that the phosphatidylinositol 3kinase/protein kinase B (PI3K/Akt) pathway plays important 
roles in mediating the multiple biological processes in tumor cells, including proliferation, apoptosis, and migration [8-11]. In addition, the PI3K/Akt pathway was demonstrated to function as a crucial pathway in the regulation of multidrug resistance (MDR) of cancer cells by modulating the activity of various MDR-related proteins, such as MDR1 and P-gp [12-14]. Recent evidence revealed that PI3K/Akt activation is strongly correlated with the inactivation of the tumor suppressor gene phosphatase and tensin homolog (PTEN) [15]. In tumor cells, at the upstream of Akt, PTEN serves as a phosphatase to block the activity of PI3K/Akt, promoting the cell proliferation, migration, and MDR [16-18]. Inactivation of PTEN is a crucial event in tumorigenesis and tumor development. Furthermore, PTEN has been identified as a new Rho-associated kinase (ROCK) substrate, and the activation of ROCK/PTEN appears to be involved in negative regulation of PI3K/Akt signaling $[19,20]$. Our previous findings suggested that activation of PP1 and PP2A contributes to ZJW-induced mitochondrial apoptosis and translocation of cofilin-1 [7]. Notably, PP1 and PP2A are negatively regulated by PI3K/Akt pathway [21, 22]. Therefore, we speculated that the mechanism of ZJW-reversed resistance to DDP may be associated with the PI3K/Akt signaling pathway, which was mediated by ROCK1/PTEN, and finally induced PP1 and PP2A mediated dephosphorylation of p-cofilin-1 and mitochondrial translocation of cofilin-1.

In the present study, in vitro as well as in vivo studies were preformed to evaluate the role of ROCK/PTEN/PI3K in ZJWreversed DDP resistance of gastric cancer. Our work may not only shed a light on the improvement of GC chemotherapy, but also provide evidence for further clinical investigation.

\section{Materials and Methods}

2.1. Cell Lines and Cultures. Human gastric cancer cell SGC7901 was provided by the Shanghai Cell Collection (Shanghai, China). Cells were cultured in RPMI-1640 medium (Gibco Laboratories, USA) containing 10\% (v/v) fetal bovine serum, $1 \%$ penicillin-streptomycin $100 \mathrm{U} / \mathrm{ml}$ penicillin, and $100 \mu \mathrm{g} / \mathrm{ml}$ streptomycin in a humidified atmosphere of $5 \% \mathrm{CO}_{2}$ in air at $37^{\circ} \mathrm{C}$.

DDP-resistant SGC7901/DDP cells were induced from SGC7901 cells, using a concentration gradient method to increase the half maximal inhibitory concentration (IC50) of DDP (as previously described) [7].

2.2. Preparation of the ZJW Extracts. Two herbs (Rhizoma Coptidis and Fructus evodiae) were from TCM pharmacy of Putuo Hospital, Shanghai University of Traditional Chinese Medicine (Shanghai, China). ZJW extracts were prepared as previously described [7].

2.3. Western Blot Analysis. Cell treated as indicated were harvested. The protein concentration was analyzed by BCA protein Assay Reagent (Sangon Biotech, Shanghai, China). Soluble lysates containing about $20 \mu \mathrm{g}$ proteins per sample were resolved with sodium dodecyl sulfate-polyacrylamide gel electrophoresis (SDS-PAGE) and then transferred onto polyvinylidene fluoride membranes. After blocking with 5\% BSA, membranes were incubated with primary antibodies at $4^{\circ} \mathrm{C}$ overnight and secondary antibodies at room temperature for $1 \mathrm{~h}$. The membrane signals were detected using an Enhanced Chemiluminescent Western Blotting Detection System (Millipore, Billerica, MA, USA) in accordance with the manufacturer's instruction. Antibodies against ROCK, PTEN, p-PI3K, PI3K, p-Akt, Akt, cofilin-1, p-cofilin-1, and GAPDH were from Cell Signaling Technology (Danvers, MA, USA). Antibodies against cleaved ROCK were purchased from Abcam (Cambridge, MA, USA). An anti-PP1 antibody was obtained from Santa Cruz Biotechnology (Dallas, TX, USA).

2.4. Cell Cycle and Apoptosis Assays. SGC-7901/DDP cells were treated as indicated. After $48 \mathrm{~h}$ of treatment, cells were collected and prepared to cell cycle and apoptosis assays. For cell apoptosis analysis, cells were stained with Annexin $\mathrm{V}$-fluorescein isothiocyanate (FITC) apoptosis detection kit (BD Biosciences, San Jose, CA, USA) and proportions of apoptotic cells were also analyzed using flow cytometry (BD Biosciences). For cell cycle analysis, cells were fixed in ethanol at $-20^{\circ} \mathrm{C}$ overnight; after washing with PBS three times, cells were stained with propidium iodide (PI, Sigma, St. Louis, $\mathrm{MO}, \mathrm{USA}$ ) at $37^{\circ} \mathrm{C}$ for $30 \mathrm{~min}$. The cell cycle distribution was assessed with flow cytometry (BD Biosciences, Franklin Lakes, NJ, USA).

2.5. Immunofluorescent Staining. SGC-7901/DDP cells 5000 per well were seeded on cover slips precoated with $0.01 \%$ poly-lysine in a 24-well chamber. After drug treatments, cells were fixed with $4 \%$ paraformaldehyde for $20 \mathrm{~min}$, permeabilized with $0.1 \%$ Triton X-100 for $10 \mathrm{~min}$, and blocked with $5 \%$ bovine serum albumin (BSA) for $60 \mathrm{~min}$ at room temperature. Cells were probed with primary antibody at $4^{\circ} \mathrm{C}$ overnight and Alexa Fluor 488-conjugated goat antirabbit IgG (Life Technologies) in the dark for $1 \mathrm{~h}$ at room temperature. After washing, images were captured with a fluorescence microscope (Leica, Wetzlar, Germany).

2.6. In Vivo Studies. A total of 36 male athymic nude mice (4-to-6-week old) were randomly divided into six groups (6 mice/group): a negative control group, a DDP group (0.6 $\mathrm{mg} / \mathrm{kg}$ ), a ZJW group (2000 mg/kg), a DDP plus $2000 \mathrm{mg} / \mathrm{kg}$ ZJW group, a DDP plus $1000 \mathrm{mg} / \mathrm{kg}$ ZJW group, and a DDP plus 500/kg ZJW group; SGC-7901/DDP cells $\left(1 \times 10^{6}\right.$ cells per mouse) were subcutaneously injected into the six groups of mice, respectively. The rats were orally administered ZJW and received intraperitoneal injection of DDP every 2 days for 4 weeks. The saline was used as control. Finally, the nude mice were killed and tumor tissues were excised and weighed.

2.7. Hematoxylin-Eosin (HE) Staining. Paraffin-embedded tissues were sliced into 4 - $\mu \mathrm{m}$-thick sections and stained with hematoxylin and eosin.

2.8. Immunohistochemistry Staining. Tumor tissues were collected, fixed with 10\% neutral-buffered formalin, dehydrated, 


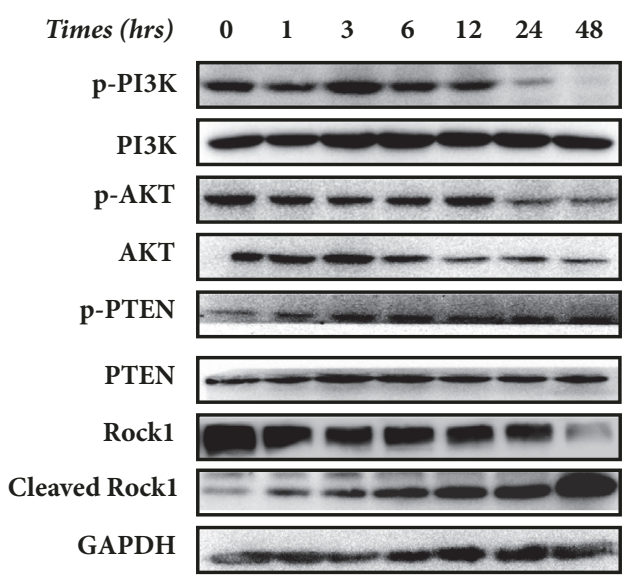

(a)

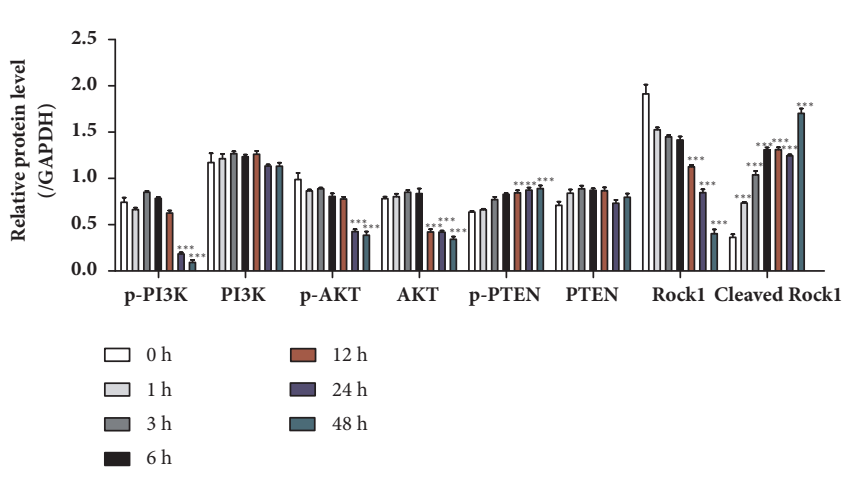

(c)

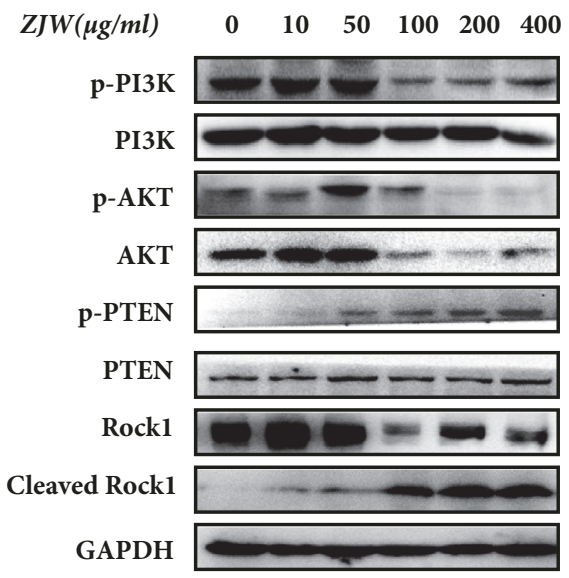

(b)

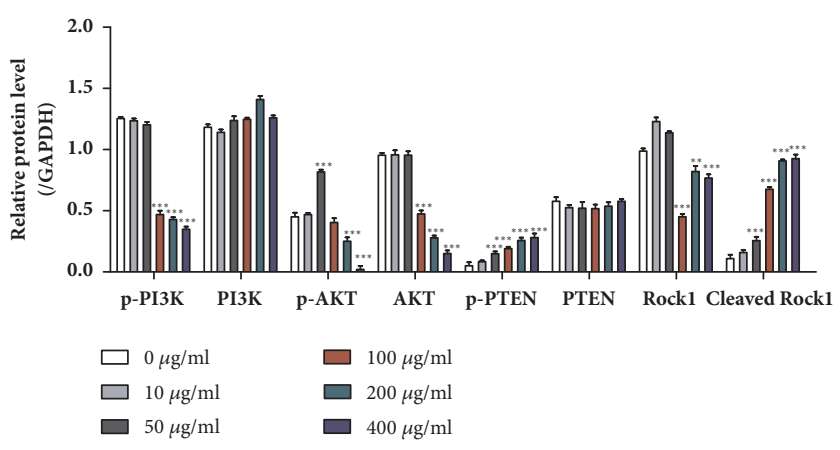

(d)

FIGURE 1: ZJW effects on ROCK/PTEN/PI3K signaling pathway in SCG-7901/DDP. (a) Cells were exposed to ZJW (50 $\mu \mathrm{g} / \mathrm{mL}$ ) for 0-48 $\mathrm{h}$. Western blotting was performed to evaluate protein levels involved in the ROCK/PTEN/PI3K pathway. (b) Cells were treated with the indicated concentration of ZJW for $48 \mathrm{~h}$; representative images of three independent western blots $(\mathrm{n}=3)$ are shown.

paraffin-embedded, and sectioned by microtome. The sections were then incubated overnight at $4^{\circ} \mathrm{C}$ with primary antip-cofilin-1, anti-cleaved ROCK, and anti-p-PTEN antibodies. HRP-conjugated anti-secondary IgG (Cell Signaling Technology) was next applied for $30 \mathrm{~min}$ at room temperature. Color was developed for $3 \mathrm{~min}$ by incubation with $3,3^{\prime}$ diaminobenzidine (Sigma). Sections were counterstained with hematoxylin and examined under microscope.

2.9. Statistical Analysis. In vivo data are presented as the mean \pm SEM and in vitro data are presented as the mean \pm SD. Group means were compared using Student's t-test or oneway ANOVA followed by Dunnett's multiple-comparison test with GraphPad Prism version 5.01 (GraphPad Software, Inc., San Diego, CA, USA). $\mathrm{P}<0.05$ was considered as statistically significant.

\section{Results}

3.1. Modulation of ZJW on ROCK/PTEN/PI3K Signaling Pathway in SGC-7901/DDP Cells. To investigate the effect of ZJW on ROCK/PTEN/PI3K pathway of gastric cancer, we examined the protein expression of ROCK/PTEN/PI3K in SGC-7901/DDP cells. SGC-7901/DDP cells were exposed to ZJW $(50 \mu \mathrm{g} / \mathrm{mL})$ for $0,1,3,6,12,24$, and $48 \mathrm{~h}$. We found that
ZJW significantly increased activity of ROCK by assessing the expression level of cleaved ROCK. To determine whether PTEN is a downstream effector of ROCK in ZJW-regulated cell apoptosis, we detected the expression of PTEN and pPTEN. The expression level of p-PTEN was upregulated in relation to control in a time dependent manner; however, the phosphorylation levels of PI3K and Akt were decreased in SGC-7901/DDP cells after ZJW 24 or $48 \mathrm{~h}$ treatment (Figure 1(a)).

Then SGC-7901/DDP cells were treated with different concentrations of ZJW $(0,10,50,100,200,400 \mu \mathrm{g} / \mathrm{mL})$ for $48 \mathrm{~h}$ (Figure 1(b)). In comparison with the control group $(0 \mu \mathrm{g} / \mathrm{mL}$ ZJW), ROCK, p-PI3K, Akt, and p-Akt expression were significantly reduced while cleaved ROCK and p-PTEN were significantly elevated in a dose-dependent manner. These results demonstrated that ZJW triggered ROCK/PTEN pathway and inhibited PI3K/Akt pathway in SGC-7901/DDP cells in a time- and dose-dependent manner.

3.2. ROCK, PI3K Inhibition Altered the Effect of ZJW in SGC7901/DDP Cells. Given that ROCK and PI3K were major regulators of cell growth and apoptosis [19], ROCK inhibitor Y27623 or PI3K inhibitor LY294002 were used to assess the potential role of ROCK/PTEN/PI3K in ZJW increased 

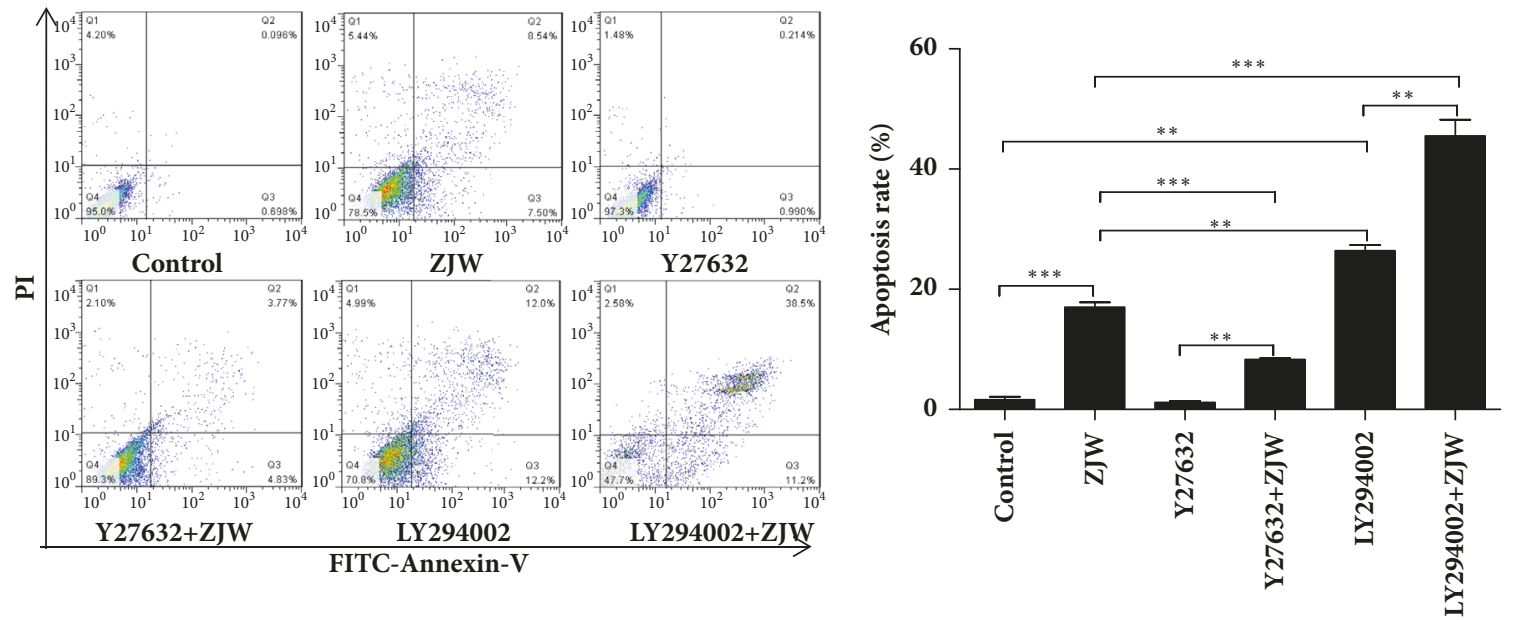

(a)
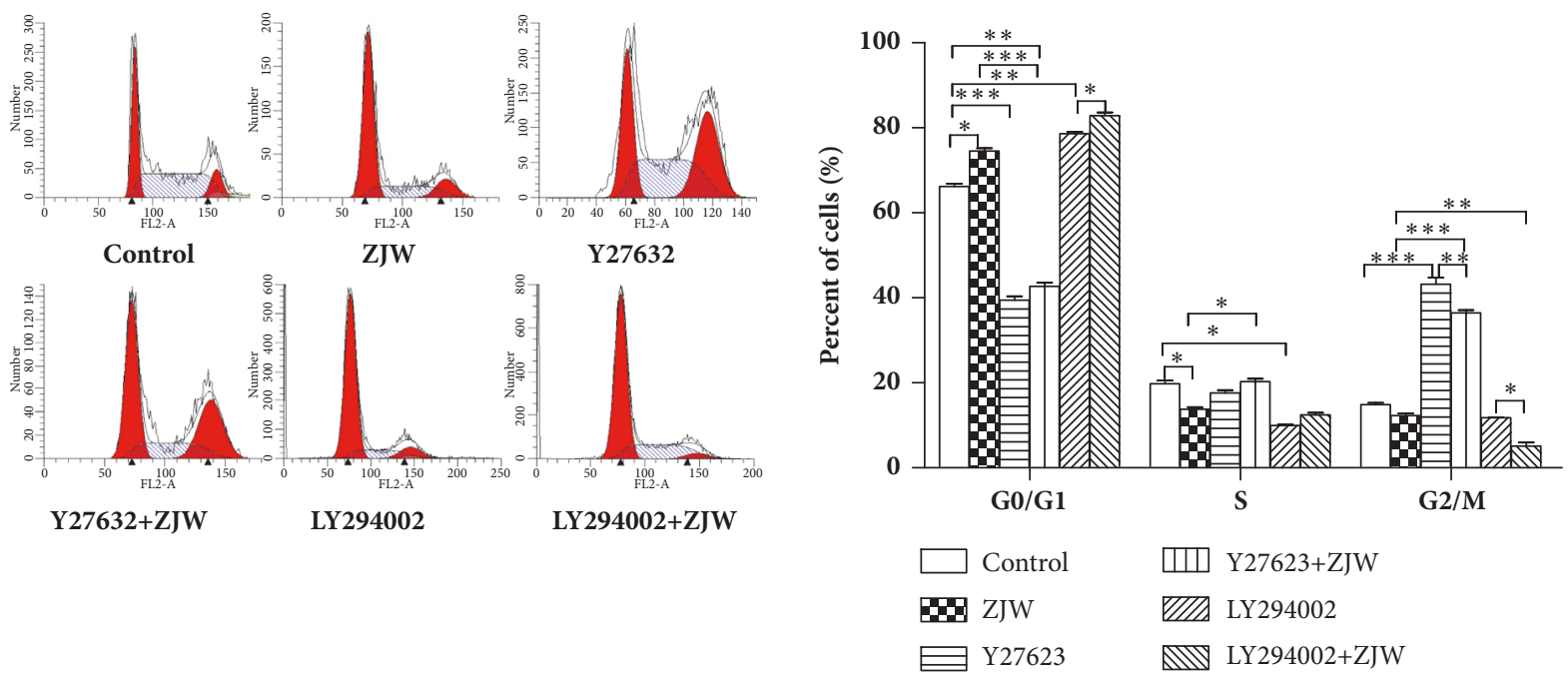

(b)

FIGURE 2: Inhibition of ROCK and PI3K effects on the apoptosis and cycle induced by ZJW. (a) Annexin V-FITC/PI double staining and flow cytometry were used to detect apoptosis in SCG-7901/DDP cells. (b) Cells were stained with PI and cell cycle distribution was analyzed by using flow cytometry. Experiments were performed three times independently. All data are represented as mean $\pm \mathrm{SD}$. ${ }^{*} \mathrm{P}<0.05$, ${ }^{* *} \mathrm{P}<$ $0.01,{ }^{* * *} \mathrm{P}<0.001$.

chemosensitivity in SGC-7901/DDP cells. As shown in Figure 2(a), ZJW significantly promoted the apoptosis of SGC7901/DDP cells. However, the combined exertion of ZJW and Y27623 yielded a significantly lower apoptosis rate than that of ZJW alone (Figure 2(a)). Furthermore, inhibition of PI3K obviously promoted ZJW-induced SGC-7901/DDP cells apoptosis. In addition, the notable increment of G2/M-phase cells was observed in SGC-7901 cells treated with ZJW and Y27623 as compared to ZJW alone (Figure 2(b)), when combined administration of ZJW and LY294002 inhibited the number of G2/M-phase phase cells. These data indicated that ROCK/PTEN/PI3K pathway contributed to the inhibitory effect of ZJW on drug resistance of SGC-7901/DDP cells.

3.3. Regulation of ROCK/PTEN/PI3K Signaling Pathway on ZJW-Mediated Dephosphorylation and Mitochondrial Translocation of Cofilin-1. We previously reported that changes in p-cofilin-1 and cofilin-1 protein expression levels were a potential mechanism of ZJW-mediated apoptosis in SGC-7901/DDP cells [7]. Therefore, we further validated whether the increased dephosphorylation and mitochondrial translocation of cofilin-1 in SGC-7901/DDP cells could be targeted by ROCK/PTEN/PI3K. We applied ZJW and ROCK inhibitor Y27623 separately and in combination, detecting the protein levels of ROCK, cleaved ROCK, pPTEN, PTEN, p-PI3K, PI3K, Akt, p-Akt, p-cofilin-1, and cofilin-1. As shown in Figures 3(a) and 3(c), ZJW significantly activated ROCK/PTEN, inhibited PI3K/Akt, and induced mitochondrial translocation of cofilin-1 in SGC7901/DDP cells, whereas Y27623 treatment had the opposite effect. In addition, the combination of ZJW and Y27623 reversed the ROCK/PTEN elevation, mitochondrial translocation of cofilin-1, and PI3K/Akt inhibition induced by ZJW. 


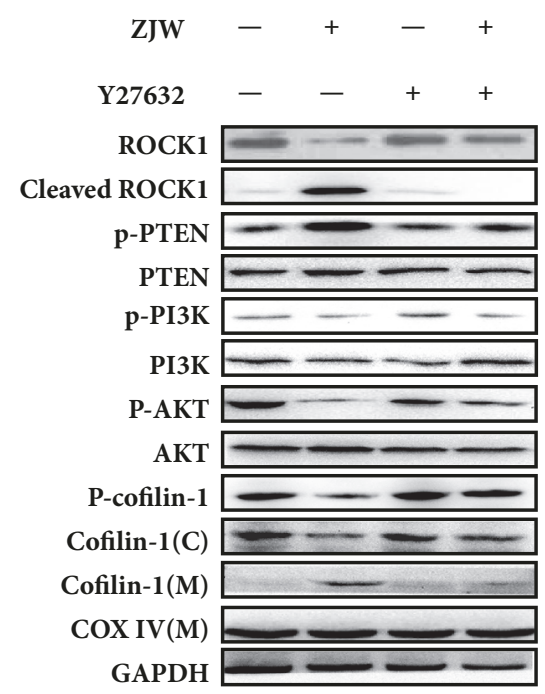

(a)

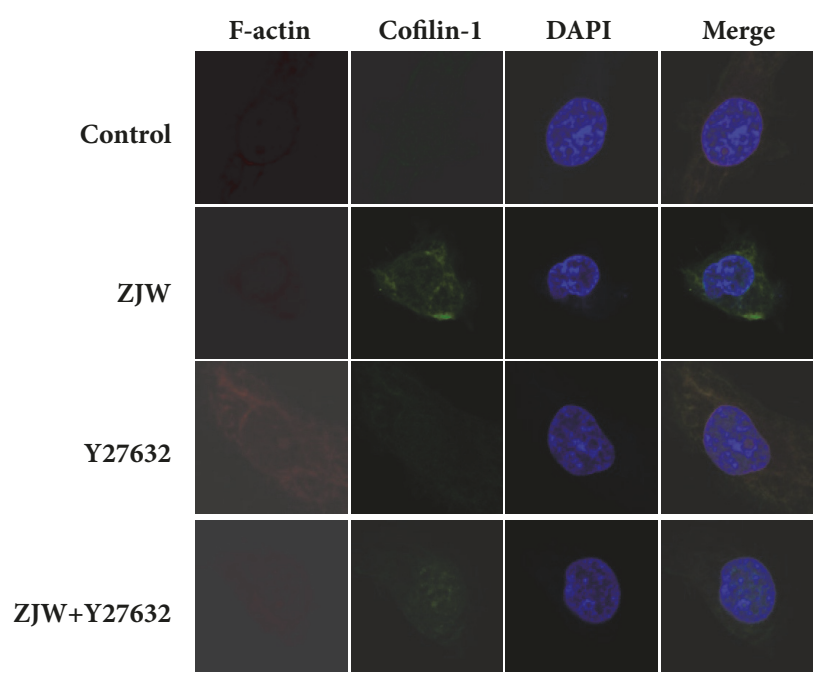

(b)
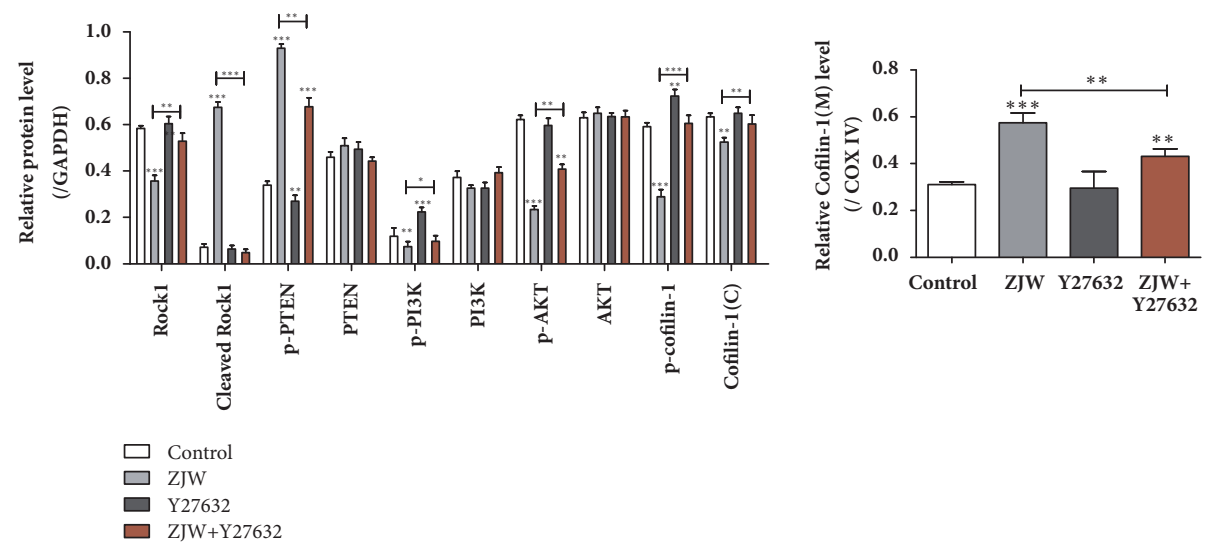

(c)

FIGURE 3: ROCK1/PTEN/PI3K signaling pathway regulates ZJW-mediated mitochondrial translocation of cofilin-1. Cells treated with ZJW (50 $\mu \mathrm{g} / \mathrm{mL})$ were cotreated with or without Y27632 for $24 \mathrm{~h}$, and western blotting was used to detect expression levels of proteins involved in the ROCK/PTEN/PI3K pathway (a). Depolymerisation of F-actin and translocation of cofilin-1 from the cytoplasm to the mitochondria in SGC7901/DDP cells were detected using an immunofluorescence assay (b). Quantification of protein levels $(\mathrm{c}) .{ }^{* *} \mathrm{P}<0.01,{ }^{* * *} \mathrm{P}<0.001$ versus control.

Immunofluorescence images (Figure 3(b)) demonstrated that ZJW treatment induced the degradation of F-actin and aggregation of cofilin-1 in the mitochondria. Y27623-treated SGC7901/DDP cells exhibited an increased expression of F-actin, while SGC7901/DDP cells treated with ZJW and Y27623 showed a significantly lower F-actin degradation and cofilin-1 accumulation in mitochondria than that observed with ZJW alone. These results indicated that the activation of ROCK played an important role in ZJW-mediated dephosphorylation of p-cofilin-1.

\subsection{PI3K Contributes to ZJW-Induced Activation of PP1/PP2A} and Mitochondrial Translocation of Cofilin-1. PI3K/Akt signaling pathway occupies a crucial position in regulating cell growth, viability, apoptosis, chemoresistance, etc. [23]. To further verify whether PI3K/Akt represented a key step in the ZJW-induced activation of PP1/PP2A and mitochondrial translocation of cofilin, the PI3K inhibitor LY294002 was performed. Western blotting analysis (Figures 4(a) and 4(c)) indicated that either ZJW or LY294002 alone could significantly repress the phosphorylation levels of PI3K and Akt and increase the activation of PP1/PP2A and mitochondrial translocation of cofilin-1. Moreover, cells cultured on both ZJW and LY294002 displayed decreased protein levels of pPI3K, p-Akt, and cofilin-1(C), while the expressions of PP1, PP2A, and cofilin-1(M) were upregulated.

Immunofluorescence analysis (Figure 4(b)) demonstrated that ZJW or LY294002 treatment significantly increased the level of F-actin. However, there was no cofilin-1 accumulation observed. ZJW and Y27623 combined exerted further enhanced F-actin expression and cofilin-1 accumulation in mitochondria than that observed with ZJW alone. These results indicated that the activation of PI3K plays an 


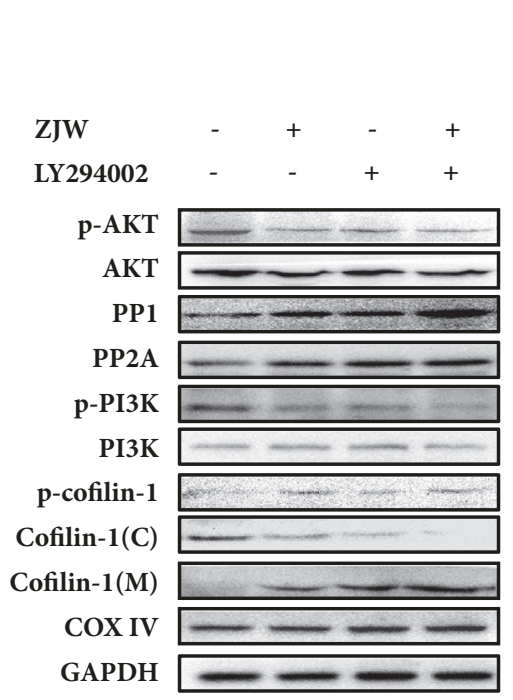

(a)

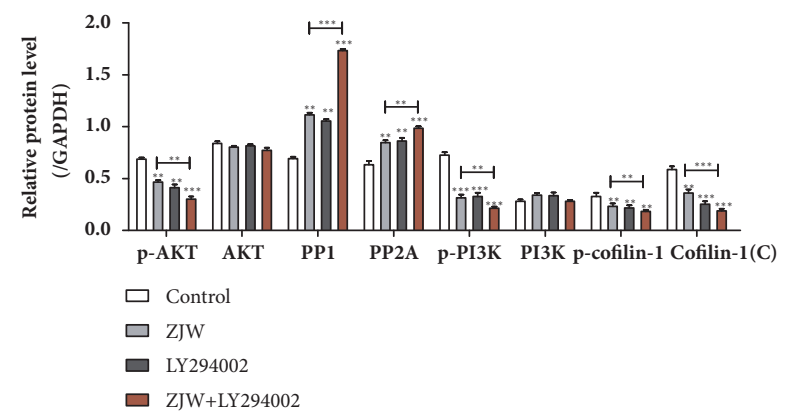

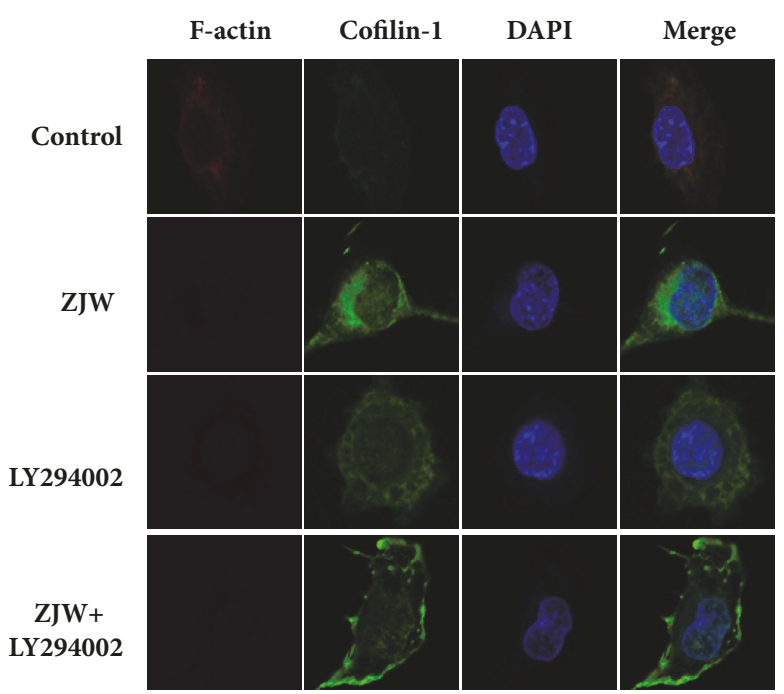

(b)

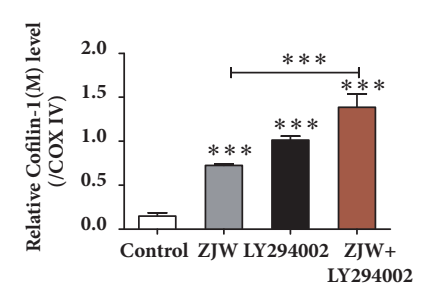

(c)

FIGURE 4: PI3K contributes to ZJW-induced activation of PP1/PP2A and mitochondrial translocation of cofilin. Cells treated with ZJW $(50 \mu \mathrm{g} / \mathrm{mL})$ were cotreated with or without LY294002 for $24 \mathrm{~h}$, and western blotting was used to detect expression levels of proteins involved in the PI3K/Akt pathway (a). Depolymerisation of F-actin and translocation of cofilin-1 from the cytoplasm to the mitochondria in SGC7901/DDP cells were detected using an immunofluorescence assay (b). Quantification of protein levels $(\mathrm{c}) .{ }^{* *} \mathrm{P}<0.01,{ }^{* * *} \mathrm{P}<0.001$ versus control.

important role in ZJW-induced activation of PP1/PP2A and mitochondrial translocation of cofilin-1.

\subsection{ZJW Inhibits Tumor Growth in SCG-7901/DDP Xenografts} Animal Model. Next, we determined whether the addition of ZJW increased the sensitivity of SCG-7901/DDP cells to DDP in vivo. Compared with control, DDP therapy exhibited significantly reduced tumor weights, but not ZJW. Mice receiving DDP and ZJW (2000 $\mathrm{mg} / \mathrm{kg}$ ) combination therapy exhibited significantly reduced tumor volumes compared with DDP alone (Figure 5). However, DDP and ZJW (1000 $\mathrm{mg} / \mathrm{kg}$ ) or DDP and ZJW $(500 \mathrm{mg} / \mathrm{kg}$ ) combination therapy did not show a significant alternation in tumor growth when compared to DDP group. It is possible that increasing the dose of ZJW may sensitize DDP-resistant cells to reach clinical efficacy. Taken together, with high dose of ZJW (2000 $\mathrm{mg} / \mathrm{kg}$ ), combination therapy could strongly suppress DDPresistant gastric cancer xenograft tumor growth.

3.6. Effects of ZJW on ROCK/PTEN /PI3K and p-Cofilin1 In Vivo. The representative $\mathrm{HE}$ staining of the indicated tumors of mice was shown in Figure 6. Our results showed that tumors treated with the combination of DDP and ZJW $(2000 \mathrm{mg} / \mathrm{kg}$ ) showed more cell vacuolization and nuclear shrinkage than with DDP alone, which was closely associated with a decrease in the tumor size in the SCG-7901/DDP xenografts mice.

Furthermore, The expression of cleaved ROCK, p-PTEN, p-PI3K, and p-cofilin-1 was assessed using immunohistochemistry to evaluate the effect of ZJW in vivo. Similar to the in vitro results, the combination of ZJW and DDP increased the expression of cleaved ROCK and p-PTEN and decreased the expression of $\mathrm{p}$-PI3K and $\mathrm{p}$-cofilin-1 compared to DDP alone. These results suggested that ZJW increased the sensitivity of DDP in GC through ROCK/PTEN/PI3K and pcofilin-1.

\section{Discussion}

DDP is a core component of chemotherapeutic treatment for gastric cancer. However, DDP resistance remains an obstacle to chemotherapy in tumor patients [24]. Our previous studies 


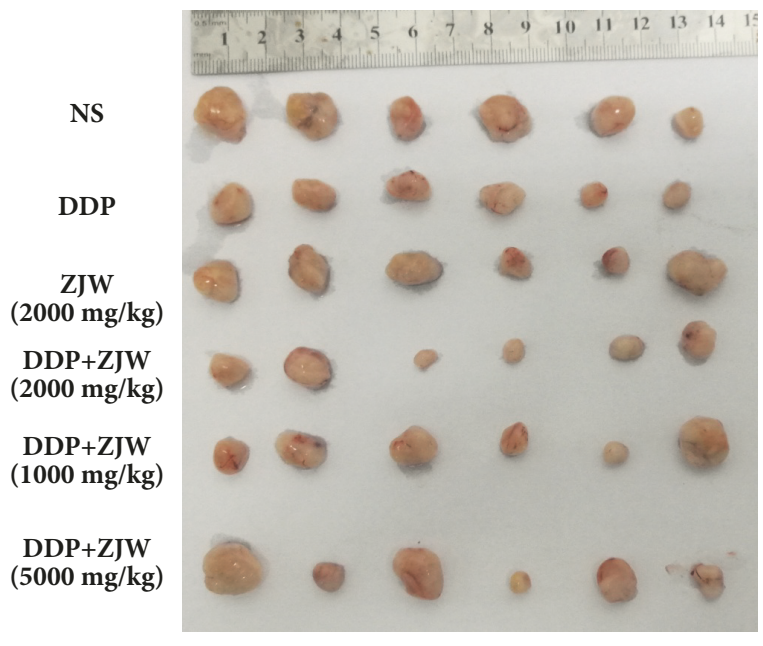

(a)

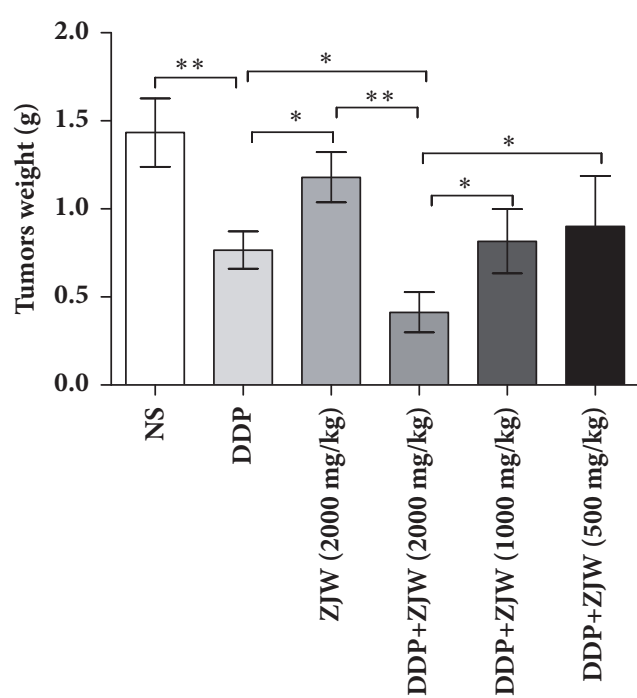

(b)

FIGURE 5: ZJW inhibits tumor growth and induces apoptosis in SCG-7901/DDP xenografts animal model. Xenograft mice were divided into six groups: negative control (NC), DDP, ZJW, DDP combined with $500 \mathrm{mg} / \mathrm{kg} \mathrm{ZJW,} \mathrm{DDP} \mathrm{combined} \mathrm{with} 1000 \mathrm{mg} / \mathrm{kg}$ ZJW, and DDP combined with $2000 \mathrm{mg} / \mathrm{kg}$ ZJW. After 30-time treatment the nude mice were killed and tumor tissues were excised and weighed. (a) Representative tumor tissues isolated from each treatment group. (b) The weight of the dissected tumors from each treatment group. Mean $\pm \mathrm{SE}, \mathrm{n}=6 .{ }^{*} \mathrm{P}<0.05,{ }^{* *} \mathrm{P}<0.01$.

have shown that a combination of DDP with the herbal extraction ZJW could cause the mitochondrial apoptosis of DDP-resistant gastric cancer cells by dephosphorylation of pcofilin-1 via activation of PP1 and PP2A [7], but the molecular mechanism remains largely unknown.

The ROCK/PTEN signaling pathway plays an important role in tumor cells apoptosis via mitochondrial translocation of cofilin-1. Activation of ROCK/PTEN could induce human prostate LNCaP cancer apoptosis by mitochondrial translocation of cofilin-1 [25]. It is clear that PI3K/Akt is essential for the development of resistance to carcinoma therapy [6] and PTEN is the main negative regulator of the PI3K/Akt pathway. Thus, the present study investigated whether the apoptosis in SCG-7901/DDP cells in response to ZJW was correlated with ROCK/PTEN/PI3K signaling pathway.

We found that ZJW had the ability to activate ROCK/ PTEN signaling pathway in a time- and dose-dependent manner, and a high level of p-PTEN antagonizes the effects of PI3K/Akt in cells. More importantly, the suppression of ROCK dramatically attenuated ZJW-induced apoptosis, anti-proliferation, and p-cofilin-1 dephosphorylation while the inhibition of PI3K had the opposite effect, determining the regulatory effects of ZJW on SCG-7901/DDP associated interactions with ROCK/PTEN/PI3K signaling pathway.

Recent studies have suggested that various mechanisms contribute to PP1 and PP2A activity [26]. PI3K was identified as a negative regulator [21, 22]. In our study, ZJW was able to inhibit PI3K and Akt. Additionally, our results revealed that PI3K inhibitor not only increased ZJW-induced apoptosis, but also enhanced expression of PP1 and PP2A and translocation of cofilin-1, suggesting that ZJW might induce activation of PP1 and PP2A in DDP-resistant gastric cells by suppressing PI3K/Akt pathway. Furthermore, accumulating literature indicated that PTEN is an important negative regulator in PI3K/Akt signaling pathway, which plays a pivotal role in cell apoptosis, growth, and proliferation [16-18], while its expression and activity can be regulated by ROCK [19]. Previous reports indicated that Rho/ROCK enhanced PTEN activity and, in the contrary, inhibited Akt activation. In MDA-MB-231 cells, the activation of RhoA/ROCK/PTEN signaling could inhibit the phosphorylation of PI3K and Akt, leading to mitochondria-mediated apoptosis [27]. Our experiment results were consistent with these reports and suggested that ROCK/PTEN activation and PI3K/Akt inhibition contributed to the cofilin-1 mitochondrial translocation and apoptosis induced by ZJW via PP1 and PP2A activation.

Preclinical in vivo evaluation of ZJW for anticancer drug resistance activity has also been conducted in subcutaneous xenograft models. A simple practice guide for dose conversion between animals and human was used to calculate the dose of ZJW and DDP used in our animal model. Consistent with the in vitro results, DDP and ZJW (2000 $\mathrm{mg} / \mathrm{kg}$ ) combination therapy significantly reduced tumor volumes compared with DDP alone (Figure 5). Moreover, the combined ZJW and DDP treatment markedly enhanced activation of ROCK and PTEN and suppressed PI3K/Akt, PP1 and PP2A activation mediated dephosphorylation of pcofilin-1, and translocation of cofilin-1 from the cytoplasm into the mitochondria.

Studies revealed that PI3K signaling pathway could be activated after DDP treatment by EGFR, which is one of the main causes of DDP treatment failure for PI3K activation and appears to be involved in several chemotherapy resistance mechanisms [28]. Therefore, PI3K/Akt inhibition seems to be a promising approach to reverse chemoresistance in cancer therapy via targeting and negatively regulating 


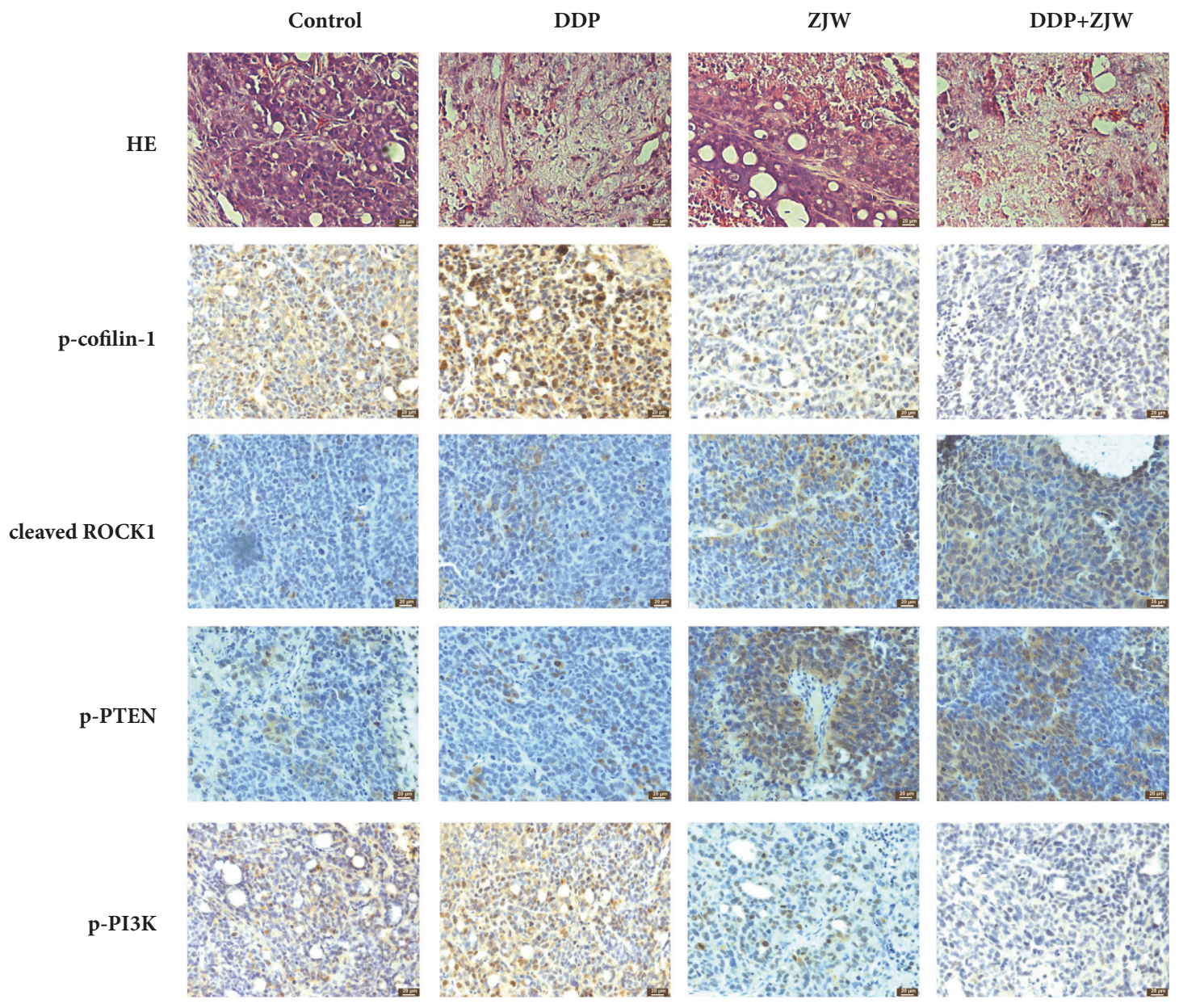

FIGURE 6: The effects of ZJW on the expression ROCK/PTEN/PI3K signaling and p-cofilin-1 of tumor bodies in vivo. (a) Representative HE staining of the indicated tumors of mice. (b) Representative photographs of IHC analysis of ROCK, PTEN, p-PI3K, and p-cofilin-1 in indicated tumors of mice.

PI3K signaling [29]. However, Phase I and II clinical trials utilizing PI3K inhibitor have shown there was no significant upregulation in survival time of advanced cancer patients [30-32]. Compared to PI3K/Akt inhibitors with serious side effects, the combination therapy using novel agents that are nontoxic, efficacious such as traditional Chinese medicine, and conventional chemotherapeutic agent like DDP significantly increased sensitization of cancer cells and reduced the toxicity of drugs via systemic regulation of multiple targets including PI3K/Akt [33-37]. Some studies have suggested that ZJW can be used as an adjuvant to many cancer therapies, improving efficacy and/or reducing adverse effects via multiple-target action like PI3K, NF-KB, and P-gp [6, $38,39]$. Thus, although the data in this study demonstrated that the ROCK/PTEN/PI3K signaling pathway plays a critical role in ZJW-induced cofilin-1 mitochondrial translocation and apoptosis via PP1 and PP2A activation, further studies are needed for future clinical use. Gastric carcinogenesis is a multistep process; Helicobacter pylori, a spiral-shaped Gramnegative bacterium, has been recognized as the causative agent for gastric cancer which was also associated with drug resistance [40-43]; multiple pharmacological effects of ZJW and its chemical constituents need to be learned to analyze its plausible role as chemopreventive agent in relation to Helicobacter pylori.

Alkaloids were proved to be the main ingredients in the treatment of digestive tract diseases [44]. Prior pharmacokinetics/pharmacodynamic studies revealed that the absorption, elimination, and systemic exposure level of these alkaloids were mainly influenced by the proportion of Coptidis Rhizoma and Evodiae Fructus, the pharmacological effect on gastrointestinal motility, and the physicochemical property of these alkaloids [45]. ZJW compatibilities reduced both Parameter apparent permeability coefficient $(\text { Papp })_{\text {basolateral } \longrightarrow \text { apical }}$ and efflux rate values of three indole alkaloids, and increased efflux rate values of two quinolone alkaloids from Evodiae Fructus [46]. Microemulsion gel delivery system can accelerate the transdermal absorption rate of ZJW, compared with the hydrogel drug delivery system, while bioavailability has no significant difference [47]. However, administration of ZJW inhibited moderately CYP2D6-mediated metabolism of dextromethorphan 
in healthy volunteers. The inhibition of CYP2D6 by ZJW could result in clinically relevant effects, either beneficial or deleterious, depending on the nature of the CYP2D6 genotype [48]. These findings would be helpful for a better understanding of the activities and clinical applications of ZJW.

\section{Conclusion}

In summary, we found that ROCK/PTEN/PI3K plays an important role in ZJW-reversed chemotherapeutic resistance of gastric cancer as a critical regulator of p-cofilin-1 dephosphorylation and mitochondrial translocation of cofilin-1. Understanding the precise role of ZJW in gastric cancer chemotherapeutic resistance and in ROCK/PTEN/PI3K signaling pathways increases our knowledge of the biological basis of cancer development and may also facilitate the development of new therapeutic strategies against gastric cancer.

$\begin{array}{ll}\text { Abbreviations } \\ \text { GC: } & \text { Gastric cancer } \\ \text { ZJW: } & \text { Zuo Jin Wan } \\ \text { TCM: } & \text { Traditional Chinese medicine } \\ \text { DDP: } & \text { Cisplatin } \\ \text { PI3K/Akt: } & \text { Phosphatidylinositol 3-kinase/protein } \\ & \text { kinase B } \\ \text { MDR: } & \text { Multidrug Resistance } \\ \text { PTEN: } & \text { Phosphatase and tensin homolog } \\ \text { ROCK: } & \text { Rho-associated kinase } \\ \text { NC: } & \text { Negative control. }\end{array}$

\section{Data Availability}

All data generated or analyzed during this study are included in this published article. Additional datasets used and/or analyzed during the current study are available from the corresponding author on reasonable request.

\section{Ethical Approval}

The use of animals in this study was approved by the Animal Research Committee in Putuo Hospital, Shanghai University of Traditional Chinese Medicine.

\section{Conflicts of Interest}

The authors declare no conflicts of interest.

\section{Authors' Contributions}

Qing-Feng Tang conceived and designed the study. Meng-Yao Sun and Jian Sun performed the experiments and collected data; Yu-Xia Yuan, Jie Tao, and Zhen-Hua Ni performed the data analysis; Meng-Yao Sun wrote the manuscript. MengYao Sun and Jian Sun contributed equally to this work. All authors discussed and interpreted the data, reviewed and edited the manuscript, and read and approved the final manuscript.

\section{Acknowledgments}

This work was supported by the National Natural Science Foundation of China (81473481) and the Health System Independent Innovation Science Foundation of Shanghai Putuo District (ptkwws201704). We give our sincere gratitude to Dr. Da-Zheng Wu who gave us so much useful advices on writing and improving the manuscript.

\section{References}

[1] T. Li, H. Liu, J. Yu, G. Shi, L. Zhao, and G. Li, "Prognostic and predictive blood biomarkers in gastric cancer and the potential application of circulating tumor cells," World Journal of Gastroenterology, vol. 24, no. 21, pp. 2236-2246, 2018.

[2] W. Chen, R. Zheng, P. D. Baade et al., "Cancer statistics in China, 2015," CA: A Cancer Journal for Clinicians, vol. 66, no. 2, pp. 115132,2016

[3] M. Orditura, G. Galizia, V. Sforza et al., "Treatment of gastric cancer," World Journal of Gastroenterology, vol. 20, no. 7, pp. 1635-1649, 2014.

[4] V. V. Subhash, S. H. Tan, W. L. Tan et al., "GTSE1 expression represses apoptotic signaling and confers cisplatin resistance in gastric cancer cells," BMC Cancer, vol. 15, article 550, 2015.

[5] J. Pan, Y. Xu, H. Song, X. Zhou, Z. Yao, and G. Ji, "Extracts of Zuo Jin Wan, a traditional Chinese medicine, phenocopies 5HTR1D antagonist in attenuating Wnt/ $\beta$-catenin signaling in colorectal cancer cells," BMC Complementary and Alternative Medicine, vol. 17, no. 1, article no. 506, 2017.

[6] H. Sui, S.-F. Pan, Y. Feng et al., "Zuo Jin Wan reverses Pgp-mediated drug-resistance by inhibiting activation of the PI3K/Akt/NF- $\kappa$ B pathway," BMC Complementary and Alternative Medicine, vol. 14, article 279, 2014.

[7] Q.-F. Tang, J. Sun, H. Yu et al., “The Zuo Jin Wan Formula Induces Mitochondrial Apoptosis of Cisplatin-Resistant Gastric Cancer Cells via Cofilin-1," Evidence-Based Complementary and Alternative Medicine, vol. 2016, Article ID 8203789, 14 pages, 2016.

[8] X. Ye, S. Cheng, Y. Dong et al., "Exendin-4 promotes proliferation of adipose-derived stem cells through PI3K/Akt-Wnt signaling pathways," Neuroscience Letters, vol. 685, pp. 196-202, 2018.

[9] Z. W. Long, W. u. JH, C. Hong, Y. N. Wang, and Y. Zhou, "MiR-374b Promotes Proliferation and Inhibits Apoptosis of Human GIST Cells by Inhibiting PTEN through Activation of the PI3K/Akt Pathway," Molecules and Cells, 2018.

[10] Y. Dong, G. Liang, B. Yuan, C. Yang, R. Gao, and X. Zhou, "MALAT1 promotes the proliferation and metastasis of osteosarcoma cells by activating the PI3K/Akt pathway," Tumor Biology, vol. 36, no. 3, pp. 1477-1486, 2015.

[11] J. Zhang, J. Xu, Y. Dong, and B. Huang, "Downregulation of HIF- $1 \alpha$ inhibits the proliferation, migration and invasion of gastric cancer by inhibiting PI3K/AKT pathway and VEGF expression," Bioscience Reports, p. BSR20180741.

[12] S. Liu, S. Chen, W. Yuan et al., "PD-1/PD-L1 interaction upregulates MDR1/P-gp expression in breast cancer cells via PI3K/AKT and MAPK/ERK pathways," Oncotarget , vol. 8, no. 59, pp. 99901-99912, 2017. 
[13] D. A. Fruman and C. Rommel, "PI3K and cancer: lessons, challenges and opportunities," Nature Reviews Drug Discovery, vol. 13, no. 2, pp. 140-156, 2014.

[14] X. Yang, Y. Ding, M. Xiao, X. Liu, J. Ruan, and P. Xue, "Antitumor compound RY10-4 suppresses multidrug resistance in MCF-7/ADR cells by inhibiting PI3K/Akt/NF- $\kappa$ B signaling," Chemico-Biological Interactions, vol. 278, pp. 22-31, 2017.

[15] A. Di Cristofano and P. P. Pandolfi, "The multiple roles of PTEN in tumor suppression," Cell, vol. 100, no. 4, pp. 387-390, 2000.

[16] Y. Chen, T. Wang, J. Du et al., "The Critical Role of PTEN/PI3K/AKT Signaling Pathway in Shikonin-Induced Apoptosis and Proliferation Inhibition of Chronic Myeloid Leukemia," Cellular Physiology and Biochemistry, vol. 47, no. 3, pp. 981-993, 2018.

[17] M. Xia, J. H. Tong, J. i. NN, M. L. Duan, Y. H. Tan, and J. G. $\mathrm{Xu}$, "Tramadol regulates proliferation, migration and invasion via PTEN/PI3K/AKT signaling in lung adenocarcinoma cells," European Review for Medical and Pharmacological Sciences, vol. 20, pp. 2573-2580, 2016.

[18] C. Lu, H. Wang, S. Chen, R. Yang, H. Li, and G. Zhang, "Baicalein inhibits cell growth and increases cisplatin sensitivity of A549 and H460 cells via miR-424-3p and targeting PTEN/PI3K/Akt pathway," Journal of Cellular and Molecular Medicine, vol. 22, no. 4, pp. 2478-2487, 2018.

[19] Z. Li, X. Dong, Z. Wang et al., "Regulation of PTEN by Rho small GTPases," Nature Cell Biology, vol. 7, no. 4, pp. 399-404, 2005.

[20] J. Chang, M. Xie, V. R. Shah et al., "Activation of Rhoassociated coiled-coil protein kinase 1 (ROCK-1) by caspase-3 cleavage plays an essential role in cardiac myocyte apoptosis," Proceedings of the National Acadamy of Sciences of the United States of America, vol. 103, no. 39, pp. 14495-14500, 2006.

[21] H. Rotfeld, P. Hillman, D. Ickowicz, and H. Breitbart, "PKA and CaMKII mediate PI3K activation in bovine sperm by inhibition of the PKC/PP1 cascade," Reproduction, vol. 147, no. 3, pp. 347356, 2014.

[22] A. Garcia, X. Cayla, J. Guergnon et al., "Serine/threonine protein phosphatases PP1 and PP2A are key players in apoptosis," Biochimie, vol. 85, no. 8, pp. 721-726, 2003.

[23] W. Shi and J. Gao, "Molecular mechanisms of chemoresistance in gastric cancer," World Journal of Gastrointestinal Oncology, vol. 8, no. 9, pp. 673-681, 2016.

[24] Z. H. Siddik, "Cisplatin: mode of cytotoxic action and molecular basis of resistance," Oncogene, vol. 22, no. 47, pp. 7265-7279, 2003.

[25] D. Mu, G. Zhou, J. Li, B. Su, and H. Guo, "Ursolic acid activates the apoptosis of prostate cancer via ROCK/PTEN mediated mitochondrial translocation of cofilin-1," Oncology Letters, vol. 15, no. 3, pp. 3202-3206, 2018.

[26] M. van Troys, L. Huyck, S. Leyman, S. Dhaese, J. Vandekerkhove, and C. Ampe, "Ins and outs of ADF/cofilin activity and regulation," European Journal of Cell Biology, vol. 87, no. 8-9, pp. 649-667, 2008.

[27] J. Zhang, L. Li, Y. Peng et al., "Surface chemistry induces mitochondria-mediated apoptosis of breast cancer cells via PTEN/PI3K/AKT signaling pathway," Biochimica et Biophysica Acta (BBA) - Molecular Cell Research, vol. 1865, no. 1, pp. 172$185,2018$.

[28] A.-M. Florea and D. Büsselberg, "Cisplatin as an anti-tumor drug: cellular mechanisms of activity, drug resistance and induced side effects," Cancers, vol. 3, no. 1, pp. 1351-1371, 2011.
[29] S. S. Singh, W. N. Yap, F. Arfuso et al., "Targeting the PI3K/Akt signaling pathway in gastric carcinoma: a reality for personalized medicine?" World Journal of Gastroenterology, vol. 21, no. 43, pp. 12261-12273, 2015.

[30] A. J. Armstrong, S. Halabi, P. Healy et al., "Phase II trial of the PI3 kinase inhibitor buparlisib (BKM-120) with or without enzalutamide in men with metastatic castration resistant prostate cancer," European Journal of Cancer, vol. 81, pp. 228236, 2017.

[31] F. Janku, "Phosphoinositide 3-kinase (PI3K) pathway inhibitors in solid tumors: From laboratory to patients," Cancer Treatment Reviews, vol. 59, pp. 93-101, 2017.

[32] M. C. Caino, J. C. Ghosh, Y. C. Chae et al., "PI3K therapy reprograms mitochondrial trafficking to fuel tumor cell invasion," Proceedings of the National Acadamy of Sciences of the United States of America, vol. 112, no. 28, pp. 8638-8643, 2015.

[33] Q.-F. Tang, Q. Ji, Y.-Y. Qiu, A.-L. Cao et al., "Synergistic Effect of Zuo Jin Wan on DDP-Induced Apoptosis in Human Gastric Cancer SGC-7901/DDP Cells," Evidence-Based Complementary and Alternative Medicine, vol. 2014, Article ID 724764, 10 pages, 2014.

[34] J. Lou, L. Yan, C. W. Bi et al., "Yu Ping Feng San reverses cisplatin-induced multi-drug resistance in lung cancer cells via regulating drug transporters and p62/TRAF6 signalling," Scientific Reports, vol. 6, no. 1, 2016.

[35] K. A. Manu, M. K. Shanmugam, L. Ramachandran et al., "Isorhamnetin augments the anti-tumor effect of capeciatbine through the negative regulation of NF- $\kappa \mathrm{B}$ signaling cascade in gastric cancer," Cancer Letters, vol. 363, no. 1, pp. 28-36, 2015.

[36] K. A. Manu, M. K. Shanmugam, L. Ramachandran et al., "First evidence that $\gamma$-tocotrienol inhibits the growth of human gastric cancer and chemosensitizes it to capecitabine in a xenograft mouse model through the modulation of NF- $\kappa \mathrm{B}$ pathway," Clinical Cancer Research, vol. 18, no. 8, pp. 2220-2229, 2012.

[37] L. Han, X. Guo, H. Bian et al., "Guizhi Fuling Wan, a Traditional Chinese Herbal Formula, Sensitizes Cisplatin-Resistant Human Ovarian Cancer Cells through Inactivation of the PI3K/AKT/mTOR Pathway," Evidence-Based Complementary and Alternative Medicine, vol. 2016, 2016.

[38] L. Xu, Y. Qi, L. Lv et al., "In vitro anti-proliferative effects of Zuojinwan on eight kinds of human cancer cell lines," Cytotechnology, vol. 66, no. 1, pp. 37-50, 2014.

[39] R. Gowda, S. V. Madhunapantula, D. Desai, S. Amin, and G. P. Robertson, "Simultaneous targeting of COX-2 and AKT using selenocoxib-1-GSH to inhibit melanoma," Molecular Cancer Therapeutics, vol. 12, no. 1, pp. 3-15, 2013.

[40] A. Sokic-Milutinovic, T. Alempijevic, and T. Milosavljevic, "Role of Helicobacter pylori infection in gastric carcinogenesis: Current knowledge and future directions," World Journal of Gastroenterology, vol. 21, no. 41, pp. 11654-11672, 2015.

[41] S. F. Zaidi, J. S. Muhammad, K. Usmanghani, and T. Sugiyama, "Pharmacological ins and outs of medicinal plants against Helicobacter pylori: A review," Pakistan Journal of Pharmaceutical Sciences, vol. 28, no. 3, pp. 1171-1176, 2015.

[42] M. Safavi, M. Shams-Ardakani, and A. Foroumadi, "Medicinal plants in the treatment of Helicobacter pylori infections," Pharmaceutical Biology, vol. 53, no. 7, pp. 939-960, 2015.

[43] M. R. Murali, S. V. Naveen, C. G. Son, and H. R. Raghavendran, "Current knowledge on alleviating Helicobacter pylori infections through the use of some commonly known natural 
products: bench to bedside," Integrative Medicine Research, vol. 3, no. 3, pp. 111-118, 2014.

[44] F. Yang, T. Zhang, R. Zhang, and Y. Ito, "Application of analytical and preparative high-speed counter-current chromatography for separation of alkaloids from Coptis chinensis Franch," Journal of Chromatography A, vol. 829, no. 1-2, pp. 137-141, 1998.

[45] P. Qian, Y. Zhang, Y. Yang, W. Xu, and X. Yang, "Pharmacokinetics Studies of 12 Alkaloids in Rat Plasma after Oral Administration of Zuojin and Fan-Zuojin Formulas," Molecules, vol. 22, no. 2, p. 214, 2017.

[46] Y.-F. Yang, Q.-L. Zhou, and X.-W. Yang, "Elucidation of Compatibility Interactions of Traditional Chinese Medicines: In Vitro Absorptions Across Caco-2 Monolayer of Coptidis Rhizoma and Euodiae Fructus in Zuojin and Fanzuojin Formulas as A Case," Phytotherapy Research, vol. 31, no. 8, pp. 1220-1229, 2017.

[47] L. Cheng, Y. Wang, S. Liu et al., "Comparative study on pharmacokinetics of six major alkaloids in Zuojin Wan microemulsion based gel and hydrogel," Zhongguo Zhongyao Zazhi, vol. 36, no. 23, pp. 3327-3331, 2011.

[48] F. Qiu, S. Liu, P. Miao et al., "Effects of the Chinese herbal formula "Zuojin Pill" on the pharmacokinetics of dextromethorphan in healthy Chinese volunteers with CYP2D6*10 genotype," European Journal of Clinical Pharmacology, vol. 72, no. 6, pp. 689-695, 2016. 


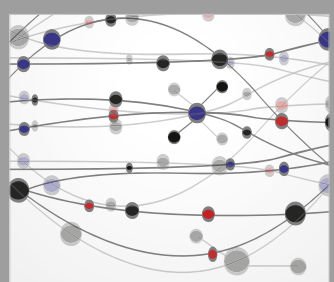

The Scientific World Journal
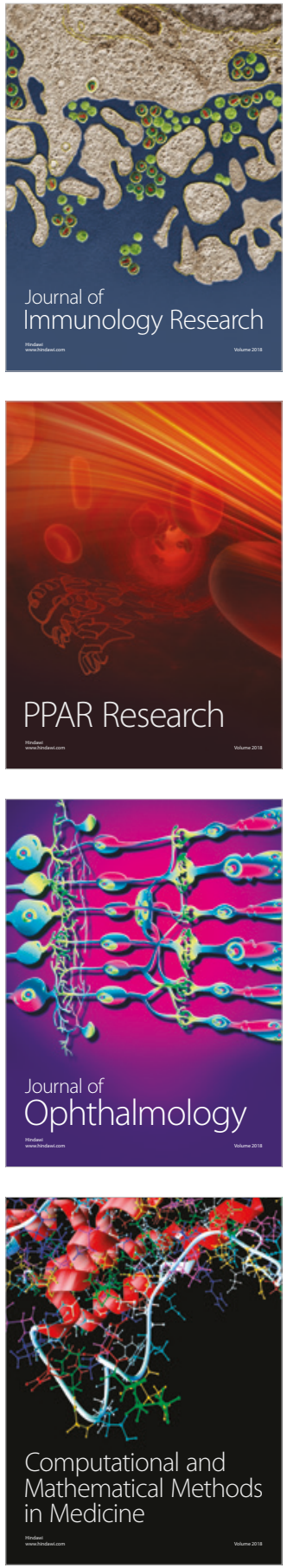

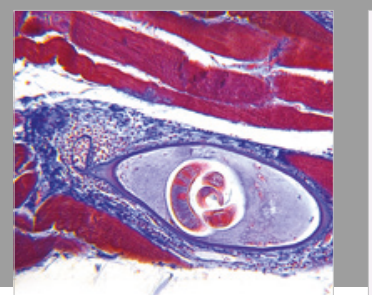

Gastroenterology Research and Practice

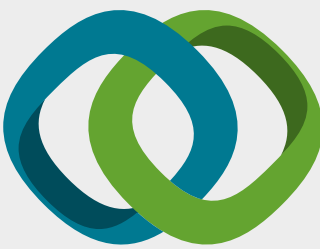

\section{Hindawi}

Submit your manuscripts at

www.hindawi.com
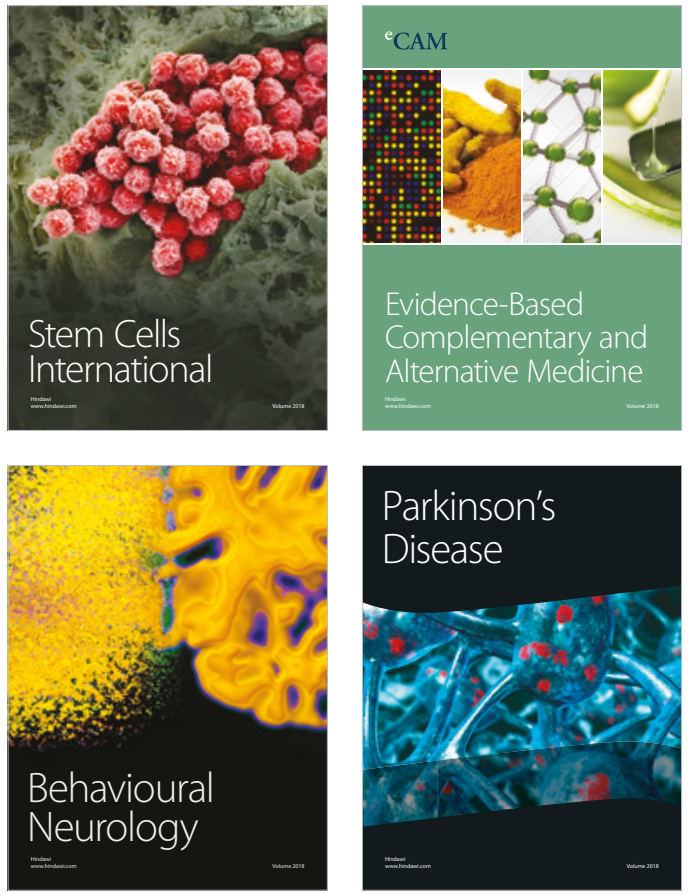

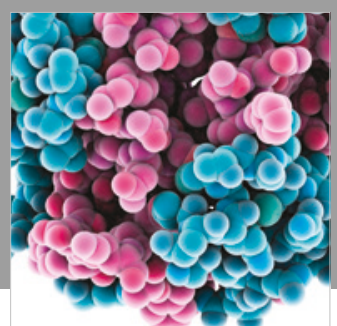

ournal of

Diabetes Research

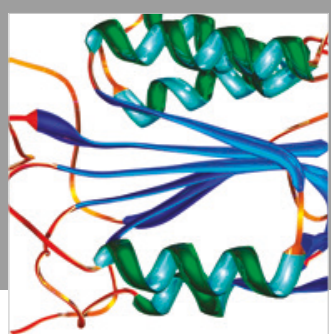

Disease Markers
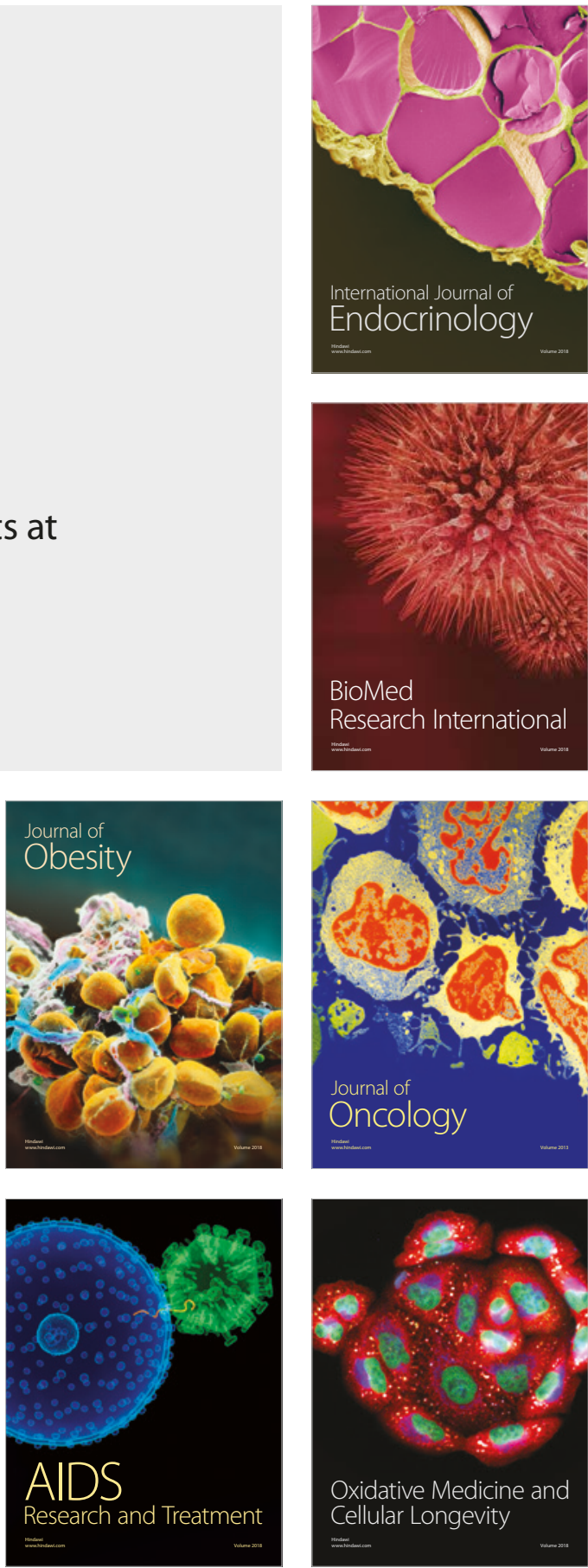